\title{
Arqueometría de materiales de construcción procedentes de Astigi
}

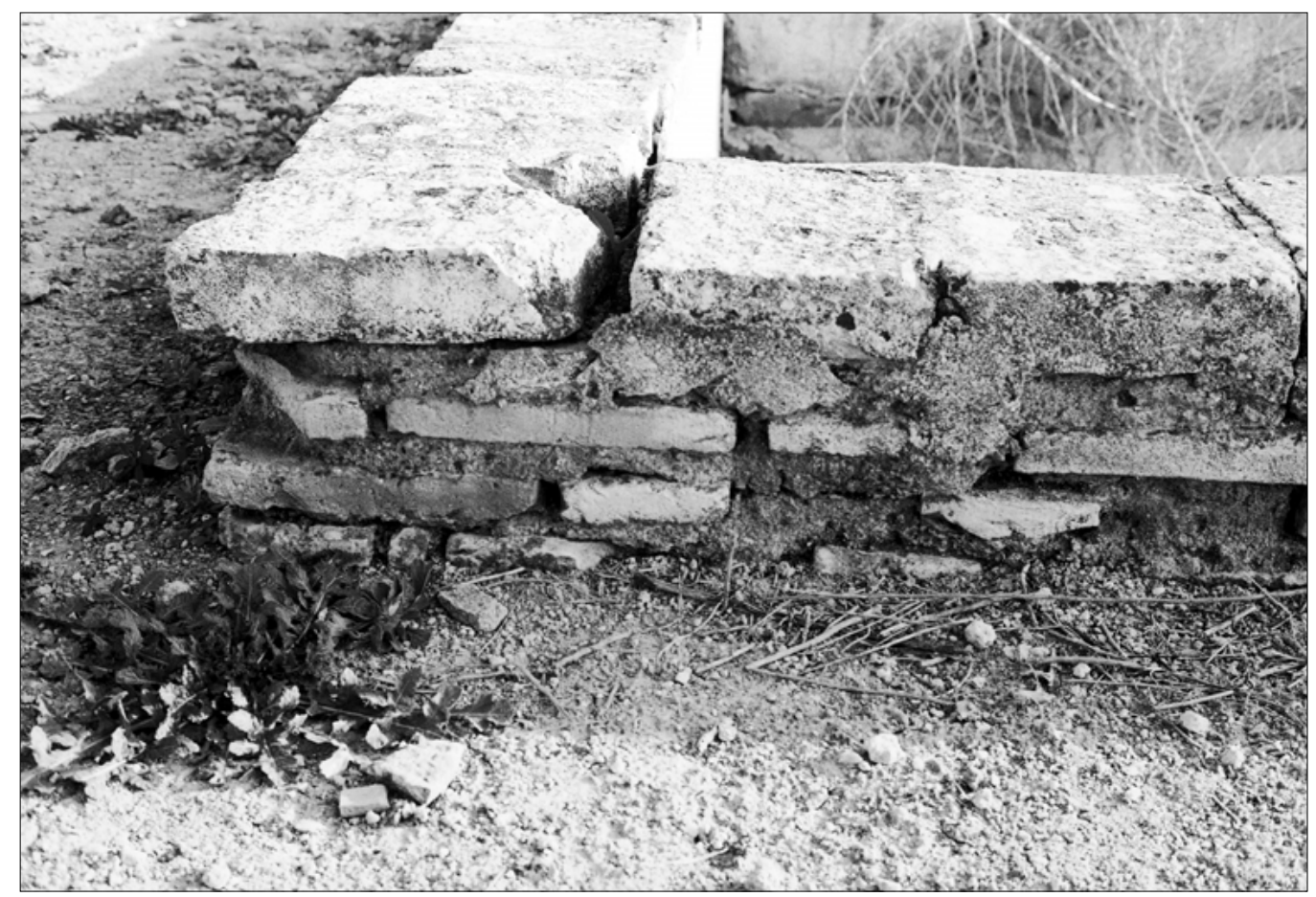

Vicente Flores Alés, Ángeles Herrera Saavedra

Dpto. Construcciones Arquitectónicas II Universidad de Sevilla

\section{Palabras clave:}

Arqueometría, Écija, ladrillo, mortero.

\section{Resumen}

En el presente trabajo se expone un resumen del estudio realizado sobre un conjunto de muestras de materiales cerámicos y morteros de época romana procedentes de excavaciones realizadas en el casco urbano de Écija y que se encontraban en el depósito de materiales arqueológicos del ayuntamiento de esta ciudad. Écija es un núcleo de población asentado sobre la que fuera ciudad romana de Astigi lo que da lugar a una gran aparición de materiales arqueológicos en cualquier excavación que se realiza. La caracterización de los materiales de construcción obtenidos permite la identificación de parámetros comunes que permiten establecer grupos de procedencia y épocas similares, de mismo modo que se reconocen las técnicas de fabricación de materiales y constructivas seguidas. 


\section{Introducción}

El estudio arqueométrico de los materiales tiene como fin establecer las características de los mismos mediante el estudio de sus parámetros físicos, composicionales,..., siguiendo diversas técnicas de caracterización ', al tiempo que se analiza el contexto histórico de los mismos. En este caso se presenta una evaluación de la procedencia de las muestras así como su caracterización física, mineralógica y química. En este trabajo se hace especial hincapié en la descripción morfológica de las muestras, pues la descripción visual de las mismas resulta una herramienta útil de cara a la clasificación de las piezas, dicha descripción no está sistematizada puesto que parte de una valoración subjetiva, pero resulta interesante ya que se produce una coherencia importante con las propiedades físicas, como así se expone más adelante.

El objetivo fundamental de este trabajo es poner de manifiesto las aportaciones que, en determinados momentos, pueden hacerse, a partir del estudio en profundidad y mayor conocimiento de los materiales, para una mejor concepción de las obras de conservación. En este marco se encuadran las actuaciones de carácter arqueológico y arqueométrico como tareas complementarias insertadas en los proyectos de restauración o rehabilitación arquitectónica, mediante estudios desde los puntos de vista histórico y científico que permiten establecer la evolución constructiva de las diferentes zonas de una edificación a lo largo del tiempo 2 .

\section{Antecedentes}

Astigi se sitúa en la ubicación actual de la ciudad de Écija, situada al nordeste de la provincia de Sevilla, en plena Campiña. Está asentada sobre una zona de materiales terciarios y cuaternarios de la cuenca del Guadalquivir. Al noroeste de la ciudad se encuentra situada una cantera de arcillas que ha sido explotada desde hace siglos para la producción alfarera, de esta cantera se han extraído con toda probabilidad las materias primas de las piezas cerámicas de época romana encontradas en la zona por ser la única de la que se tiene constancia.

Desde el punto de vista histórico, en el período que concierne a los materiales objeto de estudio, la dominación romana llega a la zona donde actualmente se asienta Écija en el siglo III a.C., en ese momento es denominada "Colonia Augusta Firma Astigi". Esta colonia, al estar situada junto a la Vía Augusta, uno de los ejes principales de comunicación en la Península, y a orillas del río Genil, se convierte en un centro neurálgico del comercio de aceite, cereales y vino; siendo cabeza de uno de los cuatro "conventus iuridici" existentes en la Bética, teniendo jurisdicción sobre todos los territorios situados al este. Las vías tenían una importancia principal en la expansión de la cultura del Imperio Romano ${ }^{3}$.

La ubicación de Astigi cumple las prescripciones indicadas por los diversos tratados existentes en la época sobre los emplazamientos humanos. Así, Catón en su tratado "De Agri Cultura" (I, 3), indica que las poblacio- nes, o en todo caso las "villae" deben estar situadas en zonas cercanas a vías de comunicación o cauces navegables, con abundancia de agua y posibilidad de comerciar con los bienes producidos. De la misma manera se expresan Columela, en el tratado "De Re Rustica" (I, 23), y Vitrubio, en "De Architectura" (I, V, I-5), haciendo hincapié ambos en la riqueza agrícola del terreno.

El transporte a través del río era el más adecuado para el comercio de aceite. Se tiene constancia de la existencia de restos de construcciones vinculadas con este comercio; así, a unos dos kilómetros del núcleo urbano de Écija se encuentran las ruinas de una fortificación para la protección del río, en el paraje conocido como "Huerta Primera del Valle", y también un pequeño "portus" a unos cinco kilómetros del pueblo, en la zona llamada "Las Delicias". Este tipo de construcciones que se citan con el término genérico de "portus", el cual no se encuentra bien definido, vienen a ser instalaciones de mantenimiento y regulación del cauce, a caballo entre puerto y esclusa. Igualmente existen restos de diversas construcciones asociadas al mantenimiento y navegabilidad del Genil 4.

A partir del siglo II d.C. se inicia la construcción de grandes obras públicas, dotando al asentamiento de sistema de cloacas, también se levanta el acueducto para el suministro de agua y un puente sobre el río Genil. Se inicia la construcción de edificios monumentales y propios de una ciudad como son termas, se supone que en número de dos, basilicas, templos, anfiteatro, que curiosamente se ubica en el actual emplazamiento de la plaza de toros, mercado y diversas "villae", este tipo de construcción es típica del campo; así como el foro, de dimensiones importantes, situado en el actual entorno de la iglesia de Santa Bárbara. La aparición de diversos mosaicos datados en el siglo II d.C. confirman, con mayor fuerza, la prosperidad de Astigi 5.

Así mismo, se establece una ordenación urbanística similar a la de los campamentos militares; este tipo de planificación fue utilizada también en otras muchas colonias del Imperio. La ordenación se basa en dos vías principales, el "cardus" en dirección norte-sur, y el "decumanus" en dirección este-oeste, estas vías partían y terminaban en las puertas de acceso a la ciudad, en Astigi estas venían a estar situadas en donde se ubican en la actualidad la Puerta Palma, la Plaza del Matadero, la Puerta de Osuna y la Plaza de Giles y Rubio. Los cuatro espacios enmarcados por las dos vías indicadas quedaban, a su vez, cruzados por calles, las cuales solían ser rectilíneas, salvo que la orografía no lo permitiese ${ }^{5}$.

Se han hallado restos de múltiples villae en la Campiña, algunas de estas ruinas hacen suponer la grandiosidad de estas edificaciones. Del mismo modo se han encontrado restos de construcciones relacionadas con la agricultura, como son silos para el almacenamiento de cereales y gran número de almazaras y molinos de aceite 6,7 .

Además de una importante industria agrícola, en Astigi se desarrolla la industria cerámica, tanto alfarera como de construcción. Se tiene constancia de la existencia de 
hornos, donde se han encontrado restos tanto de alfarería como de construcción, en el mismo núcleo del asentamiento $y$, en mayor medida, en el exterior del mismo, a orillas del río Genil, donde se tienen enumerados hasta dieciocho talleres dedicados a este oficio ${ }^{8}$. Al hecho de que se utilicen con mucha profusión los materiales cerámicos en la construcción, contribuye de forma muy especial la accesibilidad de materias primas. Igualmente se han encontrado restos de atarjeas para la conducción de agua con morteros realizados con agregado cerámico.

\section{Descripción de las muestras estudiadas}

Las muestras estudiadas se han designado con la letra E y un número, han sido en total diecinueve piezas cerámicas y dos muestras de mortero. Como se indicó anteriormente proceden mayoritariamente de catas excavadas con objeto de realizar la liberación arqueológica de solares situados en el casco urbano, las procedencias exactas de las piezas y descripción de morfologías y texturas ${ }^{9}$, son las siguientes:

\section{EI, c/ Oñate.}

Ladrillo aplantillado, con buena amasada. La presencia de una capa blanquecina e interior rojizo es común a múltiples piezas.

E2, c/ Oñate.

Ladrillo muy oscuro procedente de una columna, con forma de sector de circunferencia (1/3). Observado con lupa binocular parece contener escorias metálicas. Sus caras son muy irregulares.

E3, Puerta Cerrada.

Ladrillo roto, con amasada homogénea y con restos de mortero adherido en sus tablas, las cuales tienen una buena planeidad, con desviaciones pequeñas.

E4, Puerta Cerrada.

Ladrillo con rotura transversal a la soga, con mortero adherido en sus tablas. Presenta coqueras en su interior.

\section{E5, Puerta Cerrada}

Ladrillo con mala amasada, con grietas internas y externas. Las tablas son muy irregulares, presentando desviaciones de $5 \mathrm{~mm}$ y $7 \mathrm{~mm}$. Asimismo las aristas están bastante deterioradas.

\section{E6, Puerta Cerrada}

Ladrillo roto, con algunas coqueras de gran tamaño. Por lo demás la amasada tiene buen aspecto. Una de sus tablas es bastante irregular, con una desviación máxima de $6 \mathrm{~mm}$.

\section{E7, c/ Giles y Rubio}

Ladrillo completo, con muy buen aspecto externo. Presenta una cierta concavidad en una de sus tablas con una flecha máxima en el centro de $6 \mathrm{~mm}$.

E8, c / Giles y Rubio.

Ladrillo completo, con estrías y algunas coqueras grandes en sus caras. Las desviaciones de las tablas son considerables, en ambos casos de $6 \mathrm{~mm}$.
E9, c/ Cava n³1.

Ladrillo de gran grosor, al que le falta un trozo. Presenta grietas internas y coqueras, una de las cuales parece haber provocado la rotura. Una de sus tablas tiene una flecha de $6 \mathrm{~mm}$. Procede de una atarjea de conducción de agua.

\section{El0, cl Cava n³1.}

Ladrillo completo y con aparente buen estado, al igual que el anterior procede también de una canalización de agua.

EII, Gravera del Villar del Alcotrista.

Ladrillo en forma de cuarto de circunferencia procedente de una columna. Con buena amasada y homogeneidad en la pasta. Una de sus testas presenta una gran irregularidad, la otra, sin embargo es muy plana.

\section{EI2, c/ Secretario Armesto.}

Ladrillo roto transversalmente a la soga, presenta coqueras en su interior, así como gran número de poros de pequeño volumen. Ambas tablas presentan una gran irregularidad, alcanzando flechas de $5 \mathrm{~mm}$ y $2 \mathrm{~mm}$.

EI3, c/ Cava n², Las Delicias.

Ladrillo de pequeñas dimensiones, tipo térculli, pieza muy pequeña (soga máxima $10 \mathrm{~cm}$.) empleada para motivos decorativos y solerías. Presenta aspecto homogéneo y pequeños caliches repartidos por toda la pasta.

\section{EI4, c/ Fernando Cabada.}

Ladrillo roto transversalmente al tizón. Con gran cantidad de poros en superficie y áridos de gran tamaño. Presenta estrías en las aristas, con apariencia de ser elementos ornamentales. Tiene restos de mortero adheridos a las tablas.

EI5, c/ Rodríguez Marín.

Trozo pequeño muy irregular de ladrillo, posiblemente tipo térculli.

El6, cl Cava nl.

Ladrillo similar al anterior, térculli de pequeño tamaño. Presenta áridos grandes, así como también poros de tamaño considerable y pequeños caliches.

\section{EI7, c/ Bodegas.}

Ladrillo aplantillado, presenta coqueras y poros en toda su extensión. Aún así el aspecto de la pasta es homogéneo.

\section{EI8, c/ Padilla.}

Tégula (tipo de teja) rota, con poros internos y pequeños restos de morteros adheridos.

\section{EI9, c/ Barquete.}

Ladrillo roto cuya forma original no es reconocible, aunque una de sus caras es curva y permite suponer que formara parte de una columna, componiendo 1/3 de circunferencia, de diámetro considerable. Presenta algunas coqueras, caliches grandes y mortero adherido a sus tablas, las cuales son bastante irregulares, con flechas de $4 \mathrm{~mm}$. 


\section{Caracterización física}

El estudio de las características físicas de las piezas, debido a su origen, se ha limitado a la realización de ensayos no destructivos de acuerdo con las normas UNE vigentes para materiales de este tipo. Los ensayos realizados han sido de succión y absorción de agua, normas UNE 67-027 y 67-031 respectivamente.

Los valores que se han obtenido en los ensayos físicos son muy heterogéneos y tanto para la succión como para la absorción de agua los valores oscilan de manera significativa.

Los resultados de succión se mantienen todos dentro de los límites definidos por la normativa de calidad $\left(0,45 \mathrm{~g} / \mathrm{cm}^{2}\right)$, estos valores se pueden ver afectados por los restos adheridos de mortero, aunque de cualquier modo el comportamiento se puede definir como excelente. Entre los valores de absorción, los resultado no son tan categóricos, hay nueve que superan el máximo aceptado por la norma UNE (I8\% sobre peso seco).

Debido a las diferentes condiciones de conservación las relaciones entre unos valores y otros no son homogéneas. La observación visual de poros, coqueras y grietas permite suponer una tendencia en los resultados, sin embargo, en la mayoría de los casos, no hay correspondencia del aspecto externo, con los valores obtenidos en los ensayos.

A continuación se presentan de forma resumida los resultados obtenidos, los cuales dan idea de la calidad media de las piezas.

$\begin{array}{llll}\text { Muestras } & \text { Astigi (succión): } & & \\ \text { máximo: } \quad 0,27 & \text { mínimo: } & 0,03 \\ \text { media: } \quad 0,08 & \text { desv.std.: } & 0,13 \\ \text { Muestras } & \text { Astigi (absorción): } & & \\ \text { máximo: } \quad 22,3 & \text { mínimo: } & 7,4 \\ \text { media: } \quad 16,2 & \text { desv.std.: } & 4,4\end{array}$

\section{Caracterización mineralógica y química}

La caracterización mineralógica y química de las piezas de procedencia arqueológica puede plantearse con diversos objetivos. En unos casos permite establecer, por similitudes, grupos de una misma procedencia; determinación de rutas comerciales; de otra parte se pueden asociar las muestras a los posibles barreros originales, a partir del estudio de estos; otra posibilidad interesante es el reconocimiento de los procesos de fabricación seguidos: regularidad de las fases de trituración, amasado, secado y cocción, características del horno y temperaturas alcanzadas,...

La composición mineralógica se determinó por difracción de rayos $X$, siendo importante señalar que los valores obtenidos tienen carácter semicuantitativo y se expresan mediante rangos de porcentaje (Tabla I). Las piezas contienen mayoritariamente cuarzo y calcita, para cuyos valores se observa una gran dispersión de resultados.
Los resultados del cuarzo presentan unos valores extremos entre un $80 \%$ (EI6) y un $10 \%$ (E4) aproximadamente, de manera coherente estas muestras son las que presentan contenidos extremos de sílice. El resto de valores se agrupan mayoritariamente entorno a un 50\%.

Las muestras E5 y E3 son las que presentan los resultados más elevados de calcita estando alrededor del $50 \%$, la calcita se descompone entorno a los $750^{\circ} \mathrm{C}$, por lo que estos resultados también son coherentes con unos valores muy elevados de pérdida por calcinación a $1000^{\circ} \mathrm{C}(12,34 \%$ y $12,98 \%)$.

Salvo las muestras E2 y EI 2, en todas las demás se ha detectado la presencia de gehlenita, silicato de calcio y aluminio formado durante la cocción por encima de los $800^{\circ} \mathrm{C}$. En siete muestras se ha detectado piroxeno, posiblemente asociado a la forma diópsido, silicato de calcio y magnesio formado entorno a los $850^{\circ}-900^{\circ} \mathrm{C}$, en diversa cuantía. Estos contenidos se encuentran directamente relacionados con los valores de gehlenita, ello permite establecer los márgenes de temperatura aproximados alcanzados por las diversas piezas durante la cocción.

Con respecto a la caracterización química, cabe indicar que esta se realizó mediante análisis de componentes mayoritarios (silicio, calcio, hierro, aluminio, titanio, magnesio, manganeso, sodio y potasio) por espectroscopía de absorción atómica, salvo la silice, valorada por gravimetría. Como ya ha quedado reflejado, las piezas tienen una procedencia diversa, según se pone de manifiesto en la descripción de las muestras; a pesar de ello, no se produce una dispersión general de resultados importante en el análisis químico. Esto reafirma la presunción de un origen similar en las materias primas empleadas.

En cualquier caso, desde un primer momento se pone de manifiesto la aparición de dos grupos de muestras, claramente diferenciados, uno compuesto por las piezas El - 10 y otro por el resto. El primer grupo se diferencia del segundo por un contenido sensiblemente inferior de silicio y unos porcentajes de hierro, sodio y potasio muy superiores. El resto de parámetros analizados se comportan de manera muy similar. Un estudio de mayor profundidad permitiría establecer el origen de estas diferencias, pudiendo asociarse el mismo inicialmente a la dosificación de las materias primas.

\section{Estudio de morteros}

En el caso de los morteros, las muestras estudiadas tan sólo fueros dos, pero por lo interesante de los resultados se ha estimado conveniente la exposición de algunas circunstancias de interés. Un análisis pormenorizado de los morteros de construcción de época romana se publicó en el número 21 de $\mathrm{PH}{ }^{10}$.

Los morteros proceden de las excavaciones realizadas en las calles Santa Cruz n ${ }^{\circ} 4$ y Cava n³1, uno de ellos presenta agregado silíceo y otro, procedente de una atarjea, cerámico. En ambos casos la dosificación es de proporción 1:2, observándose sensibles diferencias en la composición debido a las diferencias de los agregados. 
Como dato más relevante cabe señalar que las curvas granulométricas de los agregados, obtenidas según las indicaciones que para arenas para morteros hace la NBE FL-90, se adaptan bastante bien a las curvas límite propuestas por la norma, el mortero realizado con arena tan sólo presenta una fracción del $7 \%$ superior al $5 \mathrm{~mm}$, mientras que el agregado cerámico viene a coincidir con la curva límite inferior, con una fracción del $3 \%$ inferior al límite para 0,063 mm y otra del I I\% superior a $5 \mathrm{~mm}$.

Estos resultados ponen de manifiesto claramente la calidad de los materiales así como el cuidado en la selección de los mismos.

\section{Conclusiones}

Las morfologías y texturas irregulares que se presentan en la descripción de las piezas resultan coherentes con los resultados obtenidos en los ensayos de absorción, estando la media muy próxima al valor límite normalizado; por contra, los resultados de succión presentan mejores valores, lo que ratifica la calidad estética y visual de las piezas señalada en la descripción de estas. A partir de la caracterización química y mineralógica se puede señalar que: a) las materias primas empleadas han tenido un tratamiento y dosificación heterogéneo reflejándose esta circunstancia en la textura de las pastas; b) las piezas han sido cocidas a temperaturas diferentes, por lo que la evolución mineralógica es variada, pudiéndose establecer grupos en rangos que van desde los $800^{\circ} \mathrm{C}$ hasta por encima de los $900^{\circ} \mathrm{C}$ y c) la presencia de carbonato de calcio en las piezas tiene mayoritariamente su origen, de acuerdo con la presencia de otras fases minerales, en la carbonatación por acción del dióxido de carbono ambiental habiéndose alcanzado grados de recarbonatación muy variables.

Asimismo es de destacar la calidad en la confección de los morteros, que independientemente de la regularidad de la dosificación se confeccionan con un agregado de muy buena calidad y características morfológicas y granulométricas.

\section{Agradecimiento}

Los autores quieren manifestar su agradecimiento al Ayuntamiento de Écija y al entonces arqueólogo municipal D. Juan Manuel Hueca, que en su momento facilitaron las muestras objeto de este estudio, así como al Departamento de Cristalografía y Mineralogía de la Universidad de Sevilla por su colaboración en los análisis mineralógicos.

Tabla 1. Análisis mineralógico

\begin{tabular}{|c|c|c|c|c|c|c|c|c|}
\hline & Q & C & $\mathbf{F}$ & H & G & D & $\mathbf{K}$ & $M$ \\
\hline EI & +++++ & ++++ & $T$ & & + & & & \\
\hline E2 & +++++ & ++ & $T$ & & & & $T$ & \\
\hline E3 & ++++ & +++++ & $T$ & & $T$ & & & \\
\hline E4 & ++ & ++++ & + & & ++ & ++ & $T$ & \\
\hline E5 & +++ & +++++ & $T$ & & + & & & \\
\hline E6 & ++++ & ++++ & + & & + & & & \\
\hline E7 & ++++ & + & ++ & $\mathrm{T}$ & + & ++ & & \\
\hline E8 & +++++ & ++ & + & & ++ & + & & $\mathrm{T}$ \\
\hline E9 & +++ & +++ & + & & ++ & + & & \\
\hline EIO & +++ & +++ & + & $\mathrm{T}$ & ++ & ++ & & \\
\hline EII & ++++ & +++ & + & & + & & & \\
\hline $\mathrm{EI} 2$ & +++++ & ++++ & $T$ & & & & & \\
\hline EI3 & +++++ & ++ & + & & + & + & & \\
\hline EI4 & +++++ & +++ & + & & + & & & \\
\hline EI5 & +++++ & ++++ & & & + & & & \\
\hline EI6 & +++++ & ++ & $\mathrm{T}$ & + & $T$ & & & \\
\hline EI7 & +++++ & ++ & + & & + & & & \\
\hline EI8 & ++++ & ++ & & & +++ & + & & $T$ \\
\hline E19 & ++++ & ++++ & $T$ & & $T$ & & & \\
\hline
\end{tabular}

Q: cuarzo, C: calcita, F: feldespatos, H: hematites, G: gehlenita, D: diópsido, K: silicato de calcio, M: mica

+++++ (muy abundante), ++++ (abundante), +++ (presente), ++ (escaso), +(indicios), t (trazas)

\section{Bibliografía}

I. FLORES-ALÉS, V., Estudio, caracterización y restauración de materiales cerámicos, IUCC-S.P. Universidad de Sevilla, 1999, pp. varias.

2. TABALES, M.A., La arqueología en los edificios históricos, Bol. I.A.P.H., n²0, sept. 1997, pp. 58-60

3. VAN NOSTRAND, J.J., The reorganisation of Spain by August, Univ. de California, P. in History IV, 1916, pp. 106.

4. ORDÓÑEZ-AGULLÓ, S., Colonia Augusta Firma Astigi, Sevilla 1988, pp. 216

5. CALDERO-BERMUDO, J.E., MÉNDEZ-VARÓ, J., Écija artística y monumental, Ed. Gráficas Sol, 1992, pp. 12-14.

6. SÁEZ, P., Agricultura Romana de la Bética I, 1987, pp. 109-185.
7. PONSICH, M., Implatation rurale antique sur le Bas-Guadalquivir II, París 1979

8. CHIC-GARCÍA, G., Epigrafía anfórica de la Bética, 1985, pp. 31 52, y Bases y desarrollo del comercio de aceite en la Bética, Tesis doctoral, Universidad de Sevilla, 1977, pp. 512-517.

9. POLVORINOS, A., HURTADO, V., GÓMEZ, A., Arqueometría cerámica del yacimiento calcolítico de Trastejón (Huelva), III Cong. Nac. Arqueometría, Sevilla, 1999

10. FLORES-ALÉS, V., GUIRAÚM, A., BARRIOS, J., Estudio de una selección de morteros de época romana en la provincia de Sevilla, Bol. I.A.P.H., n²1, Enero 1998, pp. 92-99. 